\title{
Distribuição espacial e plano de amostragem de Calacarus heveae (Acari) em seringueira
}

\author{
Noeli Juarez Ferla ${ }^{1}$, Gilberto José de Moraes $^{2} \&$ Olivier Bonato ${ }^{3}$
}

1. Pesquisador do Museu de Ciências Naturais, Centro Universitário UNIVATES, Rua Avelino Tallini, 171, 95900-000 Lajeado, RS, Brasil. (njferla@univates.br)

2. Bolsista do CNPq. Professor do Departamento de Entomologia, Fitopatologia e Zoologia Agrícola, Escola Superior de Agricultura Luiz de Queiroz (ESALQ/USP), Avenida Pádua Dias, 11, 13418-900 Piracicaba, SP, Brasil. (gjmoraes@ carpa.ciagri.usp.br)

3. Institut de Recherche pour le Développement-Centre de Biologie et de Gestion des Populations (IRD-CBGP). Campus International de Baillarget. CS 3001634988 Montferrier sur les cedex, France. (Olivier.Bonato@mpl.ird.fr)

\begin{abstract}
Spatial distribution and sampling plan for Calacarus heveae (Acari) on rubber trees. Calacarus heveae Feres, 1992 (Eriophyidae) is a mite described from specimens collected on rubber tree (Hevea brasiliensis, Euphorbiaceae) in the northwest region of the state of São Paulo. This mite prefers the adaxial face of the folioles which it can turn dry, yellowish and brownish; it can also cause leaf fall. The aim of this work was to analyze the distribution of $C$. heveae on rubber trees, to select the most representative sampling unit and to develop a sampling plan to determine the populational fluctuation. This study was conducted with clones PB 260 and IAN 873, in Itiquira and Pontes e Lacerda, respectively, both in the state of Mato Grosso. In Itiquira, significant differences were observed in four occasions in relation to the average number of mites per leaf in the different plant strata. In the samplings carried out in Pontes e Lacerda, no significant differences were observed between strata in relation to that parameter. Only in Itiquira, in one occasion, a significant difference between strata was verified in relation to the proportion of infested leaves. No significant differences were verified in relation to the average number of mites per leaf and proportion of leaves infested by $C$. heveae at different depths in the canopy. Calacarus heveae exhibits aggregated distribution in the field. To estimate the density of $C$. heveae, numeric and sampling plans were developed.
\end{abstract}

KEYWORDS. Hevea brasiliensis, mite, IPM, Eriophyidae.

RESUMO. Calacarus heveae Feres, 1992 é um eriofídeo descrito de espécimes coletados em plantas de seringueira (Hevea brasiliensis, Euphorbiaceae) na região noroeste do Estado de São Paulo. Esse ácaro prefere a face adaxial dos folíolos e pode causar a perda do brilho, amarelecimento, bronzeamento dessa região e a subseqüente queda prematura das folhas. O objetivo deste trabalho foi analisar a distribuição de $C$. heveae em seringueira, selecionar a unidade de amostragem mais representativa e desenvolver um plano de amostragem para o estudo de sua flutuação populacional. O trabalho foi conduzido com os clones PB 260 e IAN 873, respectivamente nos municípios de Itiquira e de Pontes e Lacerda, ambos no Mato Grosso. Em Itiquira, diferenças significativas foram observadas em quatro ocasiões em relação ao número médio de ácaros por folha nos diferentes estratos das plantas. Nas amostragens realizadas em Pontes e Lacerda, nenhuma diferença significativa foi encontrada entre os estratos em relação àquele parâmetro. Apenas em Itiquira, em uma ocasião de amostragem, foi verificada diferença entre os três estratos, em relação à proporção de folhas infestadas. Nenhuma diferença significativa foi verificada em relação ao número médio de ácaros por folha e proporção de folhas infestadas por $C$. heveae a diferentes distâncias da periferia da copa. Calacarus heveae exibe distribuição agregada no campo. Para estimar a densidade de $C$. heveae, um plano numérico e um plano binomial de amostragem foram desenvolvidos.

PALAVRAS-CHAVE. Hevea brasiliensis, ácaro, MIP, Eriophyidae.

Calacarus heveae Feres, 1992 foi descrita de espécimes coletados em plantas de seringueira (Hevea brasiliensis, Euphorbiaceae) na região noroeste do Estado de São Paulo. Apresenta escudo prodorsal sem setas, margens anterior e laterais reticuladas e empódio 4-raiado (FERES, 1992). Este ácaro prefere a face adaxial dos folíolos (VIEIRA \& Gomes, 1999; Ferla \& Moraes, 2002), podendo causar a perda do brilho, amarelecimento, bronzeamento e a subsequiente queda prematura das folhas (FERES, 2000). De acordo com VIEIRA \& GOMES (1999), que acompanharam a dinâmica populacional de $C$. heveae no município de Reginópolis, São Paulo, os maiores níveis populacionais ocorreram no primeiro semestre, coincidindo com o período de maior produção de látex das seringueiras. Os mesmos autores concluíram que essa espécie pode provocar níveis de desfolha acima de 75\% no clone RRIM 600. Alguns produtores afirmam que $C$. heveae pode ocasionar perdas de até $30 \%$ na produção de látex das plantas de $H$. brasiliensis (FERES, 2000).
Ainda não foi estabelecida uma metodologia de amostragem para estimar a densidade populacional ou para definir a unidade amostral de $C$. heveae em seringueira. Devido à dificuldade da contagem de todas as folhas de uma árvore, para definir a população de uma determinada espécie, é necessário definir uma unidade de amostragem que possa ser utilizada para estimar aquela população de forma confiável. O conhecimento da dinâmica populacional de uma praga é requisito para estabelecer um programa de manejo integrado. Um processo de amostragem simples, rápido e acurado é necessário para estimar a densidade populacional da praga, permitindo efetivar o seu manejo.

O objetivo deste trabalho foi analisar a distribuição de $C$. heveae nas plantas de seringueira, selecionar a unidade de amostragem mais representativa e desenvolver um plano de amostragem para o estudo de sua flutuação populacional. 


\section{MATERIAL E MÉTODOS}

Este estudo foi conduzido em campos de seringueira das Plantações Edouard Michelin Ltda., no município de Itiquira, e da Triângulo Agroindustrial S.A, no município de Pontes e Lacerda, ambos no Mato Grosso.

Em Itiquira, foi utilizado um seringal com o clone PB 260 e, em Pontes e Lacerda, o clone IAN 873, com 10 e 12 anos do transplantio, respectivamente. Essas áreas apresentavam árvores com $20 \mathrm{~m}$ de altura e densidade de 500 plantas/ha. Dez árvores foram selecionadas em cada campo, distantes pelo menos $20 \mathrm{~m}$ das bordas do seringal. As amostragens foram realizadas mensalmente, de setembro de 1998 a agosto de 1999. As folhas foram individualizadas em sacos de papel e transportadas ao laboratório para a contagem das formas móveis sob microscópio estereoscópico, anotando-se o número de indivíduos presentes nas duas faces das folhas.

Para avaliar a distribuição espacial e definir um plano de amostragem de $C$. heveae nas plantas, foram estabelecidos três estratos de copa: basal, mediano e apical. As amostras constituíram-se de nove folhas, sendo três em cada estrato e uma de cada região: distal, mediana e proximal dos ramos. As coletas foram feitas entre as linhas, no quadrante leste, onde havia maior quantidade de folhas.

Não foram aplicados agrotóxicos nos seringais e nos seis meses que antecederam a implantação deste experimento.

Distribuição de $C$. heveae na planta: seleção de uma unidade de amostragem. Para avaliar a distribuição de $C$. heveae nas plantas, foram consideradas as populações observadas na periferia dos estratos basal, mediano e apical. $\mathrm{O}$ estudo de $C$. heveae, em relação à profundidade da copa da planta, foi realizado apenas no estrato mediano da copa, nos quadrantes leste e oeste, de onde foram retiradas três folhas de cada quadrante. Essas folhas foram coletadas de ramos da periferia, posições intermediárias e próximas do caule das seringueiras.

Os resultados obtidos foram submetidos à análise de variância (ANOVA), sendo antes transformado o número médio de ácaros em $\log (\mathrm{X}+1)$ e a proporção de ácaros por estrato em arco-seno de raiz quadrada de $\mathrm{X}$ (ZAR, 1984). A proporção de folhas infestadas, por estrato, foi comparada usando o teste de $\chi^{2}$, sendo o nível de significância fixado em $\mathrm{P}<0,05$.

Nos estudos a seguir, foram usadas como unidade de amostragem as populações observadas nas três folhas coletadas na periferia de cada um dos estratos basal, mediano e apical, totalizando noventa folhas por campo.

Distribuição entre plantas. O padrão de dispersão de C. heveae no campo foi analisado pela Lei de Taylor (TAYLOR, 1961), expressa por:

$$
\mathrm{s}^{2}=\mathrm{a} \cdot \mathrm{m}^{\mathrm{b}}
$$

Essa lei relaciona a média da densidade populacional $(\mathrm{m})$ com sua variância $\left(\mathrm{s}^{2}\right)$. $\mathrm{O}$ fator " $b$ " referese ao índice de agregação, sendo $b>1, b=1$ e $b<1$, que indicam agregação, distribuição ao acaso ou distribuição regular, respectivamente. Os coeficientes "a" e "b" foram calculados através de regressão não-linear da equação da Lei de Taylor.
Correlação entre densidade populacional e proporção de folhas atacadas. WiLson \& Room (1983) incorporaram a Lei de Taylor a um modelo que descreve a relação entre a proporção de unidades de amostragem infestadas $\mathrm{P}(\mathrm{I})$ e a densidade média de ácaros em cada unidade amostral (m), como segue:

$$
\mathrm{P}(\mathrm{I})=1-\exp \left(-\mathrm{m} \cdot\left(\log _{\mathrm{e}}\left(\mathrm{a} \cdot \mathrm{m}^{\mathrm{b}-1}\right)\right) /\left(\mathrm{a} \cdot \mathrm{m}^{\mathrm{b}-1}-1\right)\right)
$$

onde "a" e "b" são os coeficientes de Taylor. Predições da densidade e de sua variância, usadas no plano binomial de amostragem subseqüente, foram transformadas de acordo com SchaAlJE et al. (1991).

Plano numérico de amostragem. WiLson \& Room (1983) incorporaram a Lei de Taylor à equação geral proposta por KARANDINOS (1976), para calcular o número mínimo de amostras $(\mathrm{N})$ a serem tomadas para calcular a densidade média $(\mathrm{m})$ com um determinado nível de confiança $\left(\mathrm{D}_{0}\right)$, expresso na seguinte proporção de médias:

$$
\mathrm{N}=\left(\mathrm{Z}_{\mathrm{a} / 2} / \mathrm{D}_{0}\right)^{2} \cdot \mathrm{a} \cdot \mathrm{m}^{\mathrm{b}-2}
$$

$\operatorname{com} \mathrm{Z}_{\mathrm{a} / 2}$ sendo o padrão normal de desvio de 1,96 e o coeficiente de confiança de 0,9 .

Plano binomial de amostragem. Para calcular o número mínimo de amostras com base na observação da presença ou ausência de ácaros em cada amostra, utilizou-se a seguinte equação proposta também por KARANDINOS (1976):

$$
\mathrm{N}=\left(\mathrm{t} / \mathrm{D}_{\mathrm{pi}_{\mathrm{i}}}\right)^{2} \cdot \mathrm{q} / \mathrm{p}
$$

onde $\mathrm{N}$ é o número de amostras necessárias para estimar a média, $\mathrm{p}=\mathrm{P}(\mathrm{I}), \mathrm{q}=1-\mathrm{p}$ e $\mathrm{D}_{\mathrm{pi}}$ é o nível de confiança que p obteve usando a equação da Lei de WILSON \& Room (1983) para achar limites de p em $m \pm D_{m}$, onde $D_{m}$ é uma proporção fixa da média.

\section{RESULTADOS E DISCUSSÃO}

Calacarus heveae mostrou uma nítida preferência pela face adaxial dos folíolos, pois poucos indivíduos foram encontrados na face abaxial. Essa preferência pela face adaxial também foi observada por FERES (2000) e FERLA \& MoraEs (2002). Somente foram encontrados indivíduos na face abaxial dos folíolos quando altas populações foram observadas na face adaxial. Por isso, para a análise dos resultados a seguir, foi utilizado o número de ácaros observados na face adaxial dos folíolos.

Distribuição de $C$. heveae na planta de seringueira. Em Itiquira, ocorreram diferenças significativas em três ocasiões em relação ao número médio de ácaros por folha, nos diferentes estratos das plantas (Fig. 1). Nos meses de novembro e dezembro de 1998 e janeiro de 1999, o estrato basal apresentou maior número de ácaros que os demais. A população do estrato basal, no início do mês de dezembro de 1998, alcançou cerca de 100 ácaros/folha, enquanto, nos demais estratos, os níveis populacionais chegaram próximo de 75 indivíduos por folha. Nos meses de setembro e outubro de 1998 e de fevereiro a maio de 1999, a infestação do ácaro foi pouco expressiva. A análise do número de ácaros observados em Pontes e Lacerda não apresentou diferença significativa entre os estratos, em relação ao número médio de ácaros por folha (Fig. 2). Nesse local, a densidade populacional dessa espécie manteve-se sempre baixa, atingindo, no máximo, quatro ácaros por folha, no estrato apical, durante o mês de dezembro de 1998. 
Em Itiquira, a proporção de ácaros presentes em cada estrato, em relação ao total de ácaros observados, foi significativamente maior no estrato basal, nas amostras coletadas em novembro de 1998 e janeiro e maio de 1999 (Fig. 3). No mês de dezembro de 1998, a proporção de ácaros foi significativamente menor no estrato basal. Em Pontes e Lacerda, não foi observada diferença significativa em relação ao número de ácaros observados nos estratos (Fig. 4). Com exceção da amostragem realizada no mês de maio de 1999, em Itiquira, não foi constatada diferença significativa em relação à proporção de folhas infestadas nos três estratos (Figs. 5, 6).
O estrato mediano não apresentou diferença significativa quanto à densidade de ácaros e à proporção de folhas infestadas em relação aos demais estratos, demonstrando ser a região da planta mais representativa no estudo da flutuação populacional de $C$. heveae.

Distribuição de $C$. heveae de acordo com a distância a partir da periferia da copa. Somente nos meses de janeiro, em Itiquira, e de março, em Pontes e Lacerda, foram encontradas diferenças significativas quanto ao número médio de ácaros e proporção de folhas infestadas por $C$. heveae em diferentes profundidades da copa das plantas (Tab. I).
Ácaros/estrato - Itiquira - MT

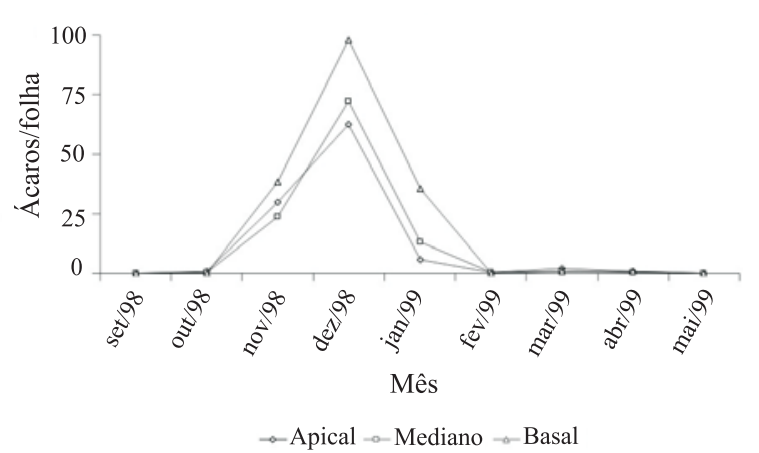

Itiquira - MT

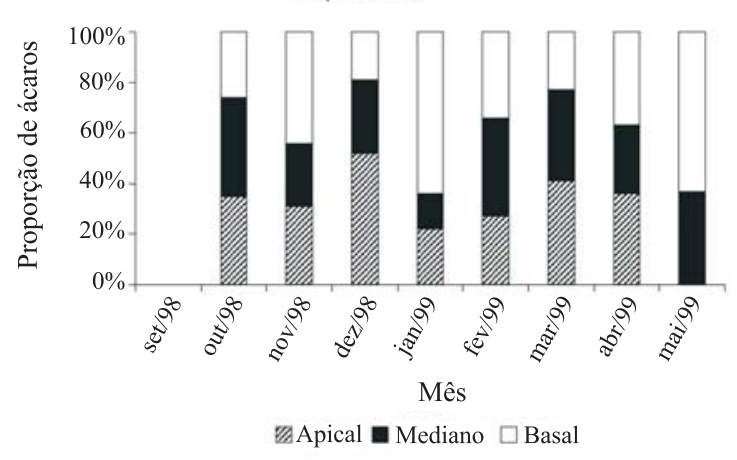

Itiquira - MT

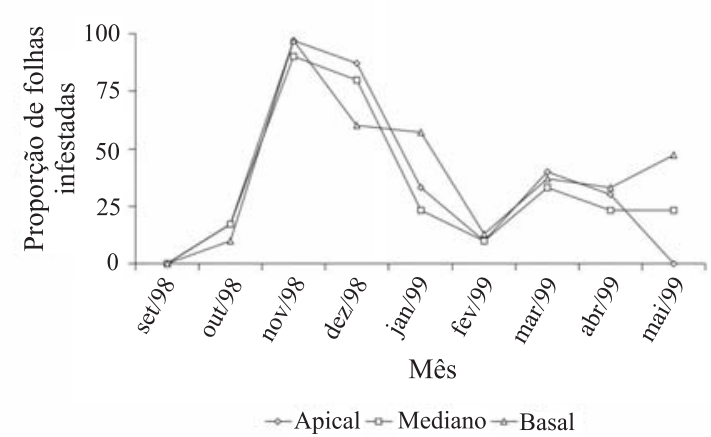

Ácaros/estrato - Pontes e Lacerda - MT

2

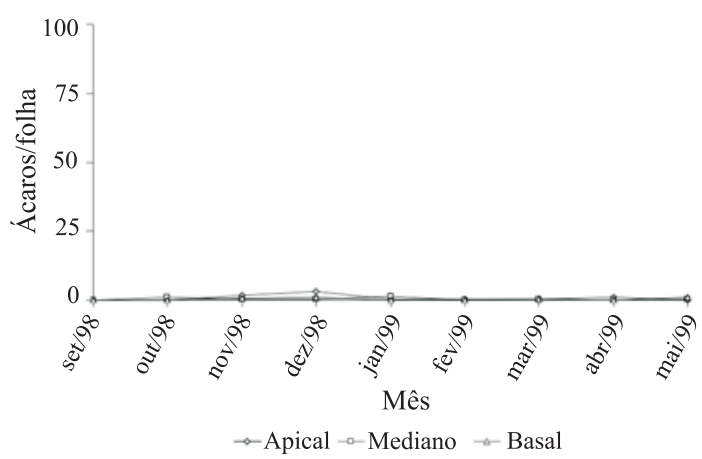

4

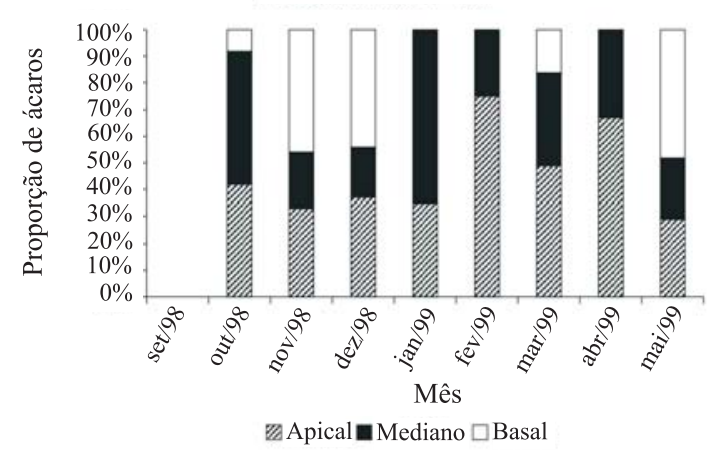

Pontes e Lacerda - MT

6

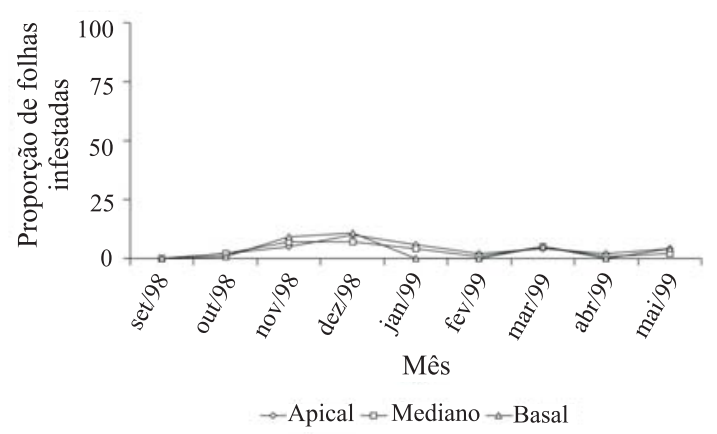

Figs. 1-6. Número médio $(1,2)$, percentagem de ácaros presentes em cada estrato $(3,4)$ e proporção de folhas infestadas $(5,6)$, por estrato em plantas de seringueira, nos municípios de Itiquira e Pontes e Lacerda, Mato Grosso. 
Distribuição entre as plantas. Os parâmetros da Lei de Taylor mostram a relação entre a variância e o número de $C$. heveae observado nas folhas do estrato médio para todas as densidades populacionais, amostragens e disposição dos espécimes nas plantas. Os valores dos parâmetros apurados foram: $\mathrm{a}=7,35 ; \mathrm{b}=1,44 ; \mathrm{R}^{2}=0,94 \mathrm{e}$ $\mathrm{gl}=14$. O parâmetro " $\mathrm{b}$ " foi significativamente maior que 1 , indicando que $C$. heveae apresenta distribuição agregada (Fig. 7).

Correlação entre densidade populacional e proporção de folhas atacadas. A curva gerada pela equação da Lei de WILSON \& Room (1983), modificada segundo SchaAlue et al. (1991), representa a relação observada no campo entre a proporção de folhas infestadas e o número médio de ácaros por folha (Fig. 8). Essa relação pode ser usada como uma estimativa preliminar da densidade populacional de $C$. heveae, a fim de definir o número de amostras a serem realizadas para expressar com segurança a população da praga no seringal. Assim, para facilitar o trabalho de amostragem no campo, pode ser observada a proporção de folhas com ácaros e estimar a média de ácaros por folha, num determinado momento.

Os resultados deste estudo demonstram a alta capacidade de dispersão de $C$. heveae na planta, uma vez que uma grande proporção de folhas estiveram infestadas mesmo quando ocorreram níveis populacionais baixos. Como exemplo, quando o nível médio de ácaros por folha era de apenas cinco ácaros, cerca de $70 \%$ das folhas dos estratos basal, mediano e apical das plantas apresentavam-se infestadas. Essa grande capacidade de colonização da planta sugere que o ácaro, provavelmente, utiliza ramos ou fissuras da epiderme da planta para a estivação. Outra possibilidade seria a presença de estímulo à migração, mesmo quando presentes baixos números de ácaros por folha.

Plano numérico de amostragem. De acordo com os resultados obtidos, para uma densidade média de 5,0 ácaros por folha e com precisão de $0,3\left(\mathrm{D}_{0}\right)$, o tamanho mínimo da amostra é de, aproximadamente, 150 folhas por campo e de, aproximadamente, 300 folhas para a precisão de 0,2 (Fig. 9).

Plano binomial de amostragem. Para uma densidade média de 5,0 ácaros por folha, o tamanho mínimo da amostra para estimar a densidade populacional de $C$. heveae, utilizando a proporção de folhas com ácaro, é de aproximadamente 50 folhas por campo para um nível de precisão de $\mathrm{D}_{0}$ de 0,3 e de cerca de 100 folhas para um nível de precisão de 0,2 (Fig. 10).

A avaliação apenas da presença ou ausência de ácaros nas folhas demonstrou ser um método mais adequado do que o numérico, pois, com menor número de folhas e em menor tempo, é possível estimar a população de $C$. heveae no seringal. Essa forma de amostragem é de fácil aplicação quando ocorre apenas uma espécie num determinado ambiente ou quando a espécie pode ser reconhecida com facilidade. Entretanto, devido à presença de mais de uma espécie de eriofídeo na cultura da seringueira (FERla \& MORAES, 2002), esse plano torna-se de difícil aplicação no campo para esta cultura. As dificuldades observadas para o plano binomial também se aplicam para o plano numérico, pois, para a contagem de ácaros, é preciso reconhecer e diferenciar os eriofídeos presentes em seringais.

Tabela I. Diferença entre o número médio de ácaros e proporção de folhas infestadas por Calacarus heveae Feres, 1992, em diferentes profundidades da copa de seringueiras, dos clones PB 260 e IAN 873, entre setembro de 1998 e maio de 1999, em Itiquira e Pontes e Lacerda, Mato Grosso (F, valor do teste ANOVA de Fisher; P, diferença entre as distâncias da periferia da copa da planta de seringueira $(\mathrm{P}<0.05)$ ).

\begin{tabular}{|c|c|c|c|c|c|c|c|c|c|}
\hline \multicolumn{10}{|c|}{ Itiquira } \\
\hline Mês/ano & $09 / 98$ & $10 / 98$ & $11 / 98$ & $12 / 98$ & $01 / 99$ & $02 / 99$ & $03 / 99$ & 04/99 & 05/99 \\
\hline $\mathrm{F}$ & 0,3 & 0,9 & 1,1 & 2,6 & 3,7 & 0,05 & 0,3 & 1,6 & 0,08 \\
\hline $\mathrm{P}$ & 0,7 & 0,4 & 0,3 & 0,7 & 0,03 & 0,9 & 0,7 & 0,2 & 0,9 \\
\hline \multicolumn{10}{|c|}{ Pontes e Lacerda } \\
\hline Mês/ano & $09 / 98$ & $10 / 98$ & $11 / 98$ & $12 / 98$ & $01 / 99$ & $02 / 99$ & 03/99 & $04 / 99$ & $05 / 99$ \\
\hline $\mathrm{F}$ & 1,4 & 0,8 & 0,2 & 0,3 & 1,0 & 2,1 & 4,2 & 2,3 & 0,5 \\
\hline $\mathrm{P}$ & 0,2 & 0,4 & 0,7 & 0,6 & 0,3 & 0,1 & 0,01 & 0,1 & 0,6 \\
\hline
\end{tabular}

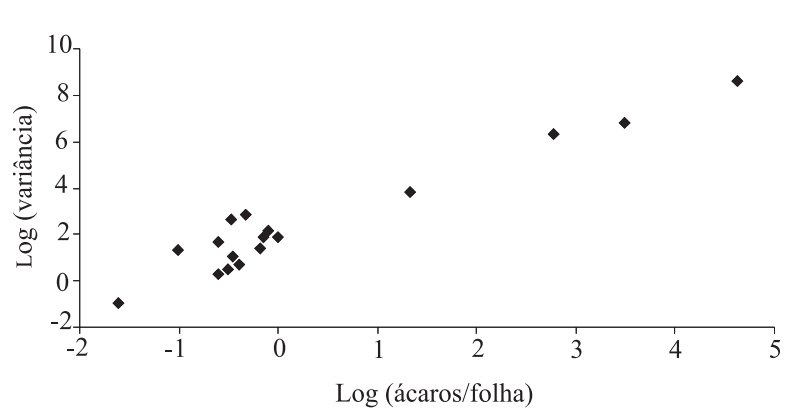

Fig. 7. Relação entre o logaritmo do número de Calacarus heveae Feres, 1992 por folha e o logaritmo de sua variância nas 180 folhas amostradas mensalmente entre setembro de 1998 e agosto de 1999 nos clones PB 260 e IAN 873 em Itiquira e Pontes e Lacerda, respectivamente.

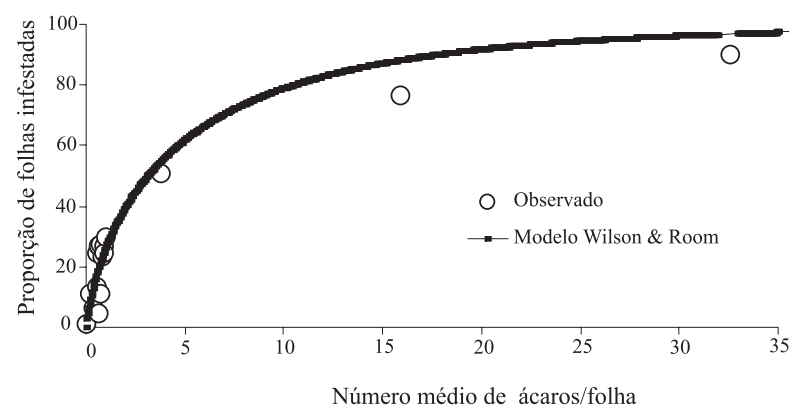

Fig. 8. Relação entre o número médio de ácaros por folha e as folhas infestadas com Calacarus heveae Feres, 1992. A curva indica a estimativa dessa relação segundo o modelo de WiLson \& Room (1983), modificado segundo SchaAlJe et al. (1991). 


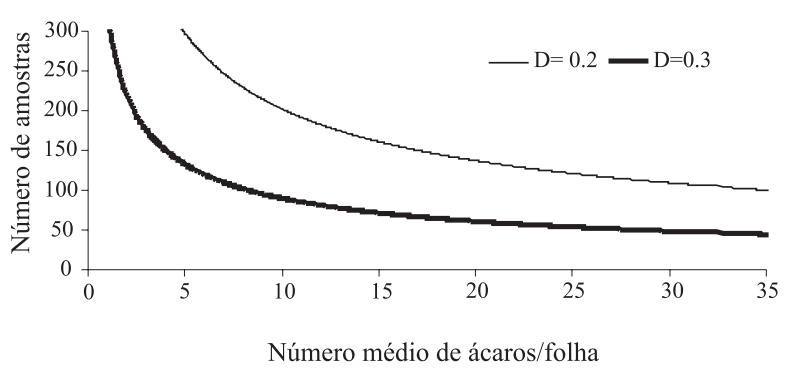

Fig. 9. Número mínimo de amostras necessárias para estimar a densidade de Calacarus heveae Feres, 1992 usando a avaliação da presença ou ausência de ácaros nas folhas para um determinado nível de precisão.

Agradecimentos. Às empresas Plantações Edouard Michellin Ltda. e Triângulo Agroindustrial Ltda., pelo financiamento do projeto. Aos Engenheiros Agrônomos Caio Franchechi, Cássio Scomparin e Etiéne Grallien, e ao Técnico Agrícola Nilson de Souza, pelo auxílio na realização destes estudos.

\section{REFERÊNCIAS BIBLIOGRÁFICAS}

Feres, R. J. F. 1992. A new species of Calacarus Keifer (Acari, Eriophyidae, Phyllocoptinae) from Hevea brasiliensis Muell. Arg. (Euphorbiaceae) from Brazil. International Journal of Acarology 18(1):61-65.

2000. Levantamento e observações naturalísticas da acarofauna (Acari, Arachnida) de seringueiras cultivadas (Hevea spp., Euphorbiaceae) no Brasil. Revista Brasileira de Zoologia 17(1):157-173.

Ferla, N. J. \& Moraes, G. J. DE. 2002. Ácaros (Arachnida, Acari) da seringueira (Hevea brasiliensis Muell. Arg.) no Estado do Mato Grosso, Brasil. Revista Brasileira de Zoologia 19(3):867-888.

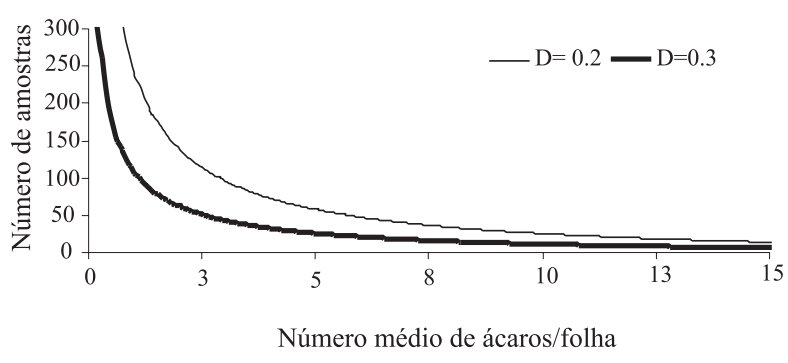

Fig. 10. Número mínimo de amostras necessárias para estimar a densidade de Calacarus heveae Feres, 1992 usando a avaliação da presença ou ausência de ácaros nas folhas para um determinado nível de precisão.

Karandinos, M. G. 1976. Optimum sample size and comments on some published formulae. Bulletin of the Entomological Society of America 22(2):417-421.

Schatue, G. B.; Butts, R. A. \& Lysyk, T. J. 1991. Simulation studies of binomial samplings: a new variance estimator and density predictor with special reference to the Russian wheat aphid (Homoptera: Aphididae). Journal of Economical Entomology 84(1): 140-147.

TAYLOR, L. R. 1961. Aggregation variance and the mean. Nature 189:732-735.

VIEIRA, M. R. \& Gomes, E. C. 1999. Sintomas, desfolhamento e controle de Calacarus heveae Feres, 1992 (Acari: Eriophyidae) em seringueira (Hevea brasiliensis Muell. Arg.). Cultura Agronômica 8(1):53-71.

Wilson, L. T. \& Room, P. M. 1983. Clumping patterns of fruit and arthropods in cotton, with implications for binomial sampling. Environmental Entomology 2(1):50-54.

ZAR, J. H. 1984. Biostatistical analysis. New Jersey, Prentice Hall. $718 \mathrm{p}$. 